\section{Tsukamurella catheter-related bloodstream infection in a pediatric patient with pulmonary hypertension}

\author{
Kristen A. Wendorf,' Claudia M. \\ Espinosa, ${ }^{2}$ William D. LeBar, ${ }^{3}$ \\ Jason B. Weinberg ${ }^{1,4}$
}

'University of Michigan Medical School; ${ }^{2}$ Department of Pediatrics and Communicable Diseases; ${ }^{3}$ Department of Pathology; and ${ }^{4}$ Department of Microbiology and Immunology, University of Michigan, Ann Arbor, Michigan, USA

\section{Abstract}

Catheter-related bloodstream infections (CR-BSI) are important complications in patients with long-term indwelling central venous catheters. In this report, we present the case of a 14-year-old male with pulmonary hypertension treated with continuous treprostinil infusion, who presented with a CR-BSI caused by a Tsukamurella species. This case highlights the potential for this unusual organism to cause infection in immunocompetent patients.

\section{Introduction}

Intravascular catheters have become an integral component of contemporary medical treatment for various diseases, and they are used commonly for infusions of chemotherapeutics and antibiotics. Complications including catheter-related bloodstream infections (CR-BSI) can be a significant source of morbidity and mortality for patients with indwelling central venous catheters. ${ }^{1}$ Patients with pulmonary hypertension require longterm central venous catheters for the continuous infusion of prostanoids, which help to reduce pulmonary artery pressure. ${ }^{2}$ Therefore, these patients are at risk for CR-BSI. While CR-BSI are frequently caused by Staphylococcus species, ${ }^{3}$ many other pathogens have been identified as causes of CR-BSI. In this report, we describe a pediatric patient with pulmonary hypertension who had a CR-BSI caused by a Tsukamurella species, which is an unusual pathogen to cause infection in an otherwise immunocompetent patient.

\section{Case Report}

A 14-year-old male with a history of idiopathic pulmonary hypertension treated with intravenous treprostinil presented to the emergency room with a three-day history of fever to $103.2^{\circ} \mathrm{F}$ along with leakage from his Broviac central venous catheter. The catheter, which had been in place for three years, was found to have an external break and subsequently was repaired. There was no evidence of infection at the insertion site. Blood cultures obtained from the catheter grew coagulase-negative staphylococci and a gram-positive bacillus. The patient was admitted to the hospital and treated with intravenous vancomycin run through the catheter. Vancomycin locks were used in the catheter between antibiotic doses.

The patient remained afebrile after admission, but a second blood culture obtained on hospital day number 2 (to determine whether the line had been sterilized) again grew a gram-positive bacillus. Catalase-positive, beaded gram-positive bacilli were isolated from subcultures to solid media. Based on these initial characteristics, a preliminary identification of a possible rapid-growing mycobacterium was considered. The patient's antibiotic regimen was changed to a combination of amikacin, clarithromycin, and linezolid on hospital day number 6 . Line removal was recommended, but the procedure was delayed until anticoagulation from the patient's longterm coumadin therapy could be reversed. On hospital day 9 , the patient became febrile for the first time after admission, although the most recent set of cultures from the line obtained from hospital days 3-8 had been negative. A transthoracic echocardiogram showed no evidence of valvular vegetations. A chest CT scan performed without contrast revealed a small left pleural effusion, scattered areas of atelectasis, and enlargement of pulmonary arteries compatible with the patient's pulmonary hypertension.

Modified acid-fast stains performed on the isolate from the first positive blood culture were negative, and the isolate was referred to the Bureau of Laboratories, at the Michigan Department of Community Health (Lansing, Michigan) for further identification. By hospital day 10 , the reference laboratory had determined that the organism was not a mycobacterium species. At that point, amikacin, clarithromycin, and linezolid were discontinued and vancomycin was started. The central venous catheter was removed on hospital day number 11. A culture of the catheter tip remained negative, but a peripheral blood culture obtained after removal of the catheter again grew a gram-positive rod. The patient remained febrile following removal of the
Correspondence: Jason B. Weinberg, 7510A MSRB I, 1150 West Medical Center Drive, Ann Arbor, Michigan 48109-5684, USA.

E-mail: jbwein@umich.edu

Key words: Tsukamurella, central venous catheter, pulmonary hypertension.

Contributions: KW, CE, WLeB, JW contributed to the conception of this project and acquisition of information regarding the case. KW drafted the original manuscript; KW, CE, WLeB, JW assisted in the revision of the manuscript, and approved the final version to be published.

Conflict of interest: the authors report no conflicts of interest.

Received for publication: 26 January 2010.

Revision received: 10 March 2010.

Accepted for publication: 11 March 2010.

This work is licensed under a Creative Commons Attribution 3.0 License (by-nc 3.0).

(C) Copyright K. Wendorf et al., 2010

Licensee PAGEPress, Italy

Infectious Disease Reports 2010; 2:e5

doi:10.4081/idr.2010.e5

catheter. No venous thrombus was identified by Doppler ultrasound evaluation of the site of his previous catheter.

Using high-performance liquid chromatography (HPLC) and other conventional biochemical tests, ${ }^{4}$ the isolate was identified as a Tsukamurella species. Based on this information, levofloxacin was added to the vancomycin therapy on hospital day 13. The patient's fever subsided quickly after this change. Multiple blood cultures (eight in total) obtained over the next 10 days remained negative. Vancomycin was discontinued on hospital day 18. A new indwelling central venous catheter was inserted on hospital day 25 . The patient remained afebrile and was discharged from the hospital shortly thereafter. Although there had been no overt evidence of endocarditis by echocardiogram or thrombus by Doppler ultrasound, the decision was made to treat the patient with an extended course of antibiotics owing to the prolonged bacteremia and the patient's underlying disease. He finished a sixweek course of oral levofloxacin with no further complications related to this infection.

The Tsukamurella isolate was sent to a second reference laboratory at National Jewish Health (Denver, Colorado) for susceptibility testing. The results (Table 1) demonstrate low minimum inhibitory concentration (MIC) values ( $<1 \mu \mathrm{g} / \mathrm{mL}$ ) for minocycline, ciprofloxacin, and trimethoprim/sulfamethoxazole. In addition, MIC values were low $(<8 \mu \mathrm{g} / \mathrm{mL})$ for amikacin, kanamycin, ceftriaxone, cefepime, imipenem, clarithromycin, and linezolid. MIC values were 
higher for tobramycin and genta-micin ( $>4$ $\mu \mathrm{g} / \mathrm{mL})$, azithromycin $(>32 / 16 \mu \mathrm{g} / \mathrm{mL})$, and amoxicillin/clavulanate $(128 \mu \mathrm{g} / \mathrm{mL})$.

\section{Discussion}

CR-BSI remains a medically important problem in patients who require long-term intravenous access. The reported incidence of CRBSI in patients with long-term indwelling central venous catheters varies from 0.3 to 9.1 infections per 1000 patient days, ${ }^{5,6}$ although higher rates have been documented in intensive care unit settings (7.7 per 1000 patient days $\left.^{6}\right)$ compared to outpatient settings ( 0.45 per 1000 patient days $s^{5}$. The risk of infection also appears to increase over time, with more infections occurring after at least 30 days of catheter use. $^{5}$

Patients receiving continuous prostanoid infusions to treat pulmonary hypertension are at risk for CR-BSI. The rate of CR-BSI in patients with pulmonary hypertension and an indwelling central venous catheter has ranged from 0.1 per 1000 patient days to 0.89 per 1000 patient days/catheter days. ${ }^{7-10}$ These rates are comparable to rates seen in other patient populations in outpatient settings. ${ }^{11,12}$ The majority of reported cases in patients with pulmonary hypertension has been caused by gram-positive organisms, especially Staphylococcus species. $^{7-10,13}$ Interestingly, both an overall increased incidence of CR-BSI and an increased proportion of CR-BSI caused by gram-negative pathogens have been reported in patients receiving treprostinil compared to patients receiving epoprostenol. ${ }^{8,9,14}$ While the presence of an indwelling central venous catheter is likely the biggest risk factor for CRBSI in this patient population, there has been some speculation that potential immunomodulatory activities of the prostanoids may increase the risk of infection. ${ }^{9}$ For instance, synthetic prostacylin analogs have been shown to inhibit bacterial phagocytosis and killing by macrophages in vitro. ${ }^{15}$

In this report, we describe a case of CR-BSI caused by a Tsukamurella species. Tsukamurella species are aerobic gram-positive organisms from the order Actinomycetales. Other genera of this order, including Nocardia, Gordonia, Streptomyces, Rhodococcus, Mycobacterium, and Corynebacterium, are closely related to Tsukamurella species; this may explain the easy misidentification of Tsukamurella in the literature. ${ }^{16}$ Tsukamurella species typically are found primarily in soil and sludge. ${ }^{17}$ Tsukamurella was first isolated in humans from the sputum of patients with chronic lung disease in 1971 by Tsukamura and Mizuno. ${ }^{18}$ The organism was known as Gordona aurantiaca ${ }^{16}$ until 1988,

Table 1. Susceptibility testing of Tsukamurella sp. isolate.

\begin{tabular}{lc} 
Antibiotic & Minimum inhibitory concentration (ug/mL) \\
Amikacin & $<8.0$ \\
Kanamycin & $<8.0$ \\
\hline Tobramycin & 4.0 \\
Gentamicin & 8.0 \\
\hline Ceftriaxone & $<8.0$ \\
Cefepime & 8.0 \\
\hline Cefotaxime & 16.0 \\
Imipenem & 4.0 \\
\hline Minocycline & $<1.0$ \\
Ciprofloxacin & $<1.0$ \\
\hline Clarithromycin & 2.0 \\
Azithromycin & 128.0 \\
\hline Trimethoprim/Sulfamethoxazole & $<0.5 / 9.5$ \\
Amoxicillin/Clavulanate & $>32 / 16$ \\
\hline Linezolid & 4.0 \\
\hline
\end{tabular}

when it was first proposed as a genus based on gene sequence analysis. ${ }^{19}$ Seven Tsukamurella species have been identified, but only five have been reported to cause infections in humans, including T. inchonensis, T. paurometabola, T. pulmonis, T. strandjordae, and T. tyrosinosolvens. The first case of bacteremia caused by $T$. pulmonis was reported in 1998 in an immunosuppressed patient. ${ }^{20} T$. pulmonis was identified subsequently as a cause of cavitary pneumonia and CR-BSI in immunocompromised patients. ${ }^{21}$ T. tyrosinosolvens has been identified in a patient with gastric carcinoma who developed hemoptysis ${ }^{22}$ and in immunocompromised patients with CR-BSI, ${ }^{23}$ as well as in an immunocompetent patient with a brain abscess. ${ }^{24}$ T. pulmonis and T. tyrosinosolvens have also been implicated in conjunctivitis in immunocompetent patients. ${ }^{25}$ Finally, T. paurometabola has been isolated in immunosuppressed patients with CR-BSI. ${ }^{26}$

Tsukamurella species are infrequent causes of CR-BSI. In a recent review, Bouza and colleagues noted that 12 of 14 reported patients with CR-BSI caused by Tsukamurella species were being treated for an underlying malignancy. ${ }^{27}$ The remaining patients had chronic renal failure and required long-term intravenous access for hemodialysis. In general, underlying conditions in patients presenting with Tsukamurella CR-BSI have included primary immunodeficiencies, a variety of malignancies, and chronic renal failure..$^{16,23,26-29}$ One case of Tsukamurella CR-BSI in a patient receiving intravenous epoprostenol for pulmonary hypertension was included in a recent series of CR-BSI in patients with pulmonary hypertension, ${ }^{8}$ although no details of the case presentation are included in the report. Although it is possible that our patient's treprostinil therapy and underlying lung disease increased his risk for CR-BSI, the break in his central venous catheter was the most likely risk factor for developing CR-BSI caused by both the Tsukamurella species and the coagulase-negative staphylococcus.

Tsukamurella species may be misidentified as atypical Mycobacterium species when conventional mycobacterial testing is used initially in the identification of a gram-positive bacillus. ${ }^{30,31}$ Given the difficulty of identifying Tsukamurella isolates using routine laboratory techniques, it is likely that some cases of Tsukamurella CR-BSI have been incorrectly attributed to other pathogens. Of the Tsukamurella species associated with CR-BSI reviewed here, five were first identified as Corynebacterium,,$^{16,23,27-29}$ four as Nocardia, ${ }^{26,27,29}$ two as Rhodococcus ${ }^{23,26}$ one as Bacillus, ${ }^{29}$ and one as a nontuberculous mycobacterium. ${ }^{16}$ Final identification as Tsukamurella species often depended on the use of techniques that are not readily available in most clinical microbiology laboratories, such as $\mathrm{HPLC}^{16,26}$ or $16 \mathrm{~S}$ rRNA sequencing. ${ }^{16,23,26,27}$

In vitro testing suggests that Tsukamurella isolates may be susceptible to quinonlones, macrolids, carbapenams, trimethoprim/sulfamethoxazole, and aminoglycosides..$^{16,23,27,28}$ Low MIC values for ciprofloxacin, imepenam, and trimethoprim/sulfamethoxazole (Table 1) suggest that this was true for the isolate that we describe in this report. The activity of other classes of antibiotics varies in other reports. Susceptibilities may vary even within a class of antibiotics, as with the aminoglycosides for the isolate described here (Table 1). Importantly, antibiotics that are common empiric choices when a gram-positive rod is isolated may not have sufficient activity against Tsukamurella species. For instance, although MIC values for vancomycin were not 
available for the isolate obtained from our patient, other reports have documented MIC values for vancomycin between 2 and 8 $\mu \mathrm{g} / \mathrm{mL}^{27}$ Many antibiotics have been used to treat Tsukamurella CR-BSI, particularly trimethoprim/sulfamethoxazole and quinolones by themselves or in combination with other antibiotics. ${ }^{16,27}$ Because of the varying susceptibilities of Tsukamurella species and the relative lack of clinical experience with Tsukamurella CR-BSI, appropriate identification of a Tsukamurella species and subsequent susceptibility testing are therefore essential to facilitate appropriate antimicrobial therapy. In the setting of CR-BSI caused by a Tsukamurella species, treatment in our patient and in all other previously reported cases $^{16,20,23,27,28}$ ultimately required the removal of the infected catheter in addition to antimicrobial therapy.

In summary, it is important to consider Tsukamurella species in cases of CR-BSI, particularly when specific identification of a gram-positive bacillus is difficult or delayed or when a patient fails to respond promptly to empiric antibiotic therapy. Our report highlights the potential for Tsukamurella species, while most often associated with CR-BSI in immunocompromised patients, to cause disease in other patient populations including otherwise immunocompetent patients treated with intravenous prostanoids for pulmonary hypertension.

\section{References}

1. O'Grady NP, Alexander M, Dellinger EP, et al. Guidelines for the prevention of intravascular catheter-related infections. Infect Control Hosp Epidemiol 2002;23: 759-69.

2. McLaughlin VV, Shillington A, Rich S. Survival in primary pulmonary hypertension: the impact of epoprostenol therapy. Circulation 2002;106:1477-82.

3. Wisplinghoff $\mathrm{H}$, Bischoff T, Tallent SM, et al. Nosocomial bloodstream infections in US hospitals: analysis of 24,179 cases from a prospective nationwide surveillance study. Clin Infect Dis 2004;39:309-17.

4. Brown JM, McNeil MM. Media and methods for the identification of aerobic actinomycetes. In: Clinical Microbiology Procedures Handbook. 2nd ed. Isenberg HD, ed. Washington, D.C.: ASM Press, 2004, p 6.2.1-6.2.11.

5. Moureau N, Poole S, Murdock MA, et al. Central venous catheters in home infusion care: outcomes analysis in 50,470 patients. J Vasc Interv Radiol 2002;13:1009-16.

6. Pronovost P, Needham D, Berenholtz S, et al. An intervention to decrease catheterrelated bloodstream infections in the ICU. N Engl J Med 2006;355:2725-32.

7. Akagi S, Matsubara H, Ogawa A, et al. Prevention of catheter-related infections using a closed hub system in patients with pulmonary arterial hypertension. Circ J 2007;71:559-64.

8. Ivy DD, Calderbank M, Wagner BD, et al. Closed-hub systems with protected connections and the reduction of risk of catheter-related bloodstream infection in pediatric patients receiving intravenous prostanoid therapy for pulmonary hypertension. Infect Control Hosp Epidemiol 2009;30:823-9.

9. Kallen AJ, Lederman E, Balaji A, et al. Bloodstream infections in patients given treatment with intravenous prostanoids. Infect Control Hosp Epidemiol 2008; 29:342-9.

10. Oudiz RJ, Widlitz A, Beckmann XJ, et al. Micrococcus-associated central venous catheter infection in patients with pulmonary arterial hypertension. Chest 2004; 126:90-4.

11. Lafrance JP, Iqbal S, Lelorier J, et al. Vascular access-related bloodstream infections in First Nations, Community and Teaching Canadian Dialysis Units, and other centre-level predictors. Nephron Clin Pract 2009;114:c204-12.

12. Weber DJ, Brown V, Huslage $\mathrm{K}$, et al. Device-related infections in home health care and hospice: infection rates, 19982008. Infect Control Hosp Epidemiol 2009; 30:1022-4.

13. Decker MD, Edwards KM. Central venous catheter infections. Pediatr Clin North Am 1988;35:579-612.

14. CDC. Bloodstream infections among patients treated with intravenous epoprostenol or intravenous treprostinil for pulmonary arterial hypertension--seven sites, United States, 2003-2006. Morb Mortal Wkly Rep 2007;56:170-2.

15. Aronoff DM, Peres CM, Serezani CH, et al. Synthetic prostacyclin analogs differentially regulate macrophage function via distinct analog-receptor binding specificities. J Immunol 2007;178:1628-34.

16. Schwartz MA, Tabet SR, Collier AC, et al. Central venous catheter-related bacteremia due to Tsukamurella species in the immunocompromised host: a case series and review of the literature. Clin Infect Dis 2002;35:e72-7.

17. McNeil MM, Brown JM. The medically important aerobic actinomycetes: epidemiology and microbiology. Clin Microbiol Rev 1994;7:357-417.

18. Tsukamura M, Mizuno S. [A new species Gordona aurantiaca occurring in sputa of patients with pulmonary disease]. Kekkaku 1971;46:93-8.

19. Collins MD, Smida J, Dorsch M, et al. Tsukamurella generus novel harboring Corynebacterium paurometabolum and Rhodococcus aurantiacus. Int Journal Syst Bacteriol 1988;38:385-91.

20. Maertens J, Wattiau P, Verhaegen J, et al. Catheter-related bacteremia due to Tsukamurella pulmonis. Clin Microbiol Infect 1998;4:51-3.

21. Perez VA, Swigris J, Ruoss SJ. Coexistence of primary adenocarcinoma of the lung and Tsukamurella infection: a case report and review of the literature. $\mathrm{J}$ Med Case Reports 2008;2:207.

22. Matsumoto T, Shiraishi M, Yoshimura H, et al. [Tsukamurella tyrosinosolvens cultured from sputum of a patient who received total gastrectomy for gastric cancer]. Kekkaku 2006;81:487-90.

23. Elshibly S, Doherty J, Xu J, et al. Central line-related bacteraemia due to Tsukamurella tyrosinosolvens in a haematology patient. Ulster Med J 2005;74:43-6.

24. Sheng WH, Huang YT, Chang SC, et al. Brain abscess caused by Tsukamurella tyrosinosolvens in an immunocompetent patient. J Clin Microbiol 2009;47:1602-4.

25. Woo PC, Ngan AH, Lau SK, et al. Tsukamurella conjunctivitis: a novel clinical syndrome. J Clin Microbiol 2003;41: 3368-71.

26. Shapiro CL, Haft RF, Gantz NM, et al. Tsukamurella paurometabolum: a novel pathogen causing catheter-related bacteremia in patients with cancer. Clin Infect Dis 1992;14:200-3.

27. Bouza E, Perez-Parra A, Rosal M, et al. Tsukamurella: a cause of catheter-related bloodstream infections. Eur J Clin Microbiol Infect Dis 2009;28:203-10.

28. Jones RS, Fekete T, Truant AL, et al. Persistent bacteremia due to Tsukamurella paurometabolum in a patient undergoing hemodialysis: case report and review. Clin Infect Dis 1994;18:830-2.

29. Sheridan EA, Warwick S, Chan A, et al. Tsukamurella tyrosinosolvens intravascular catheter infection identified using $16 \mathrm{~S}$ ribosomal DNA sequencing. Clin Infect Dis 2003;36:e69-70.

30. Almuzara MN, De Mier C, Rodriguez CR, et al. [Evaluation of API Coryne System, version 2.0, for diphteroid gram-positive rods identification with clinical relevance]. Rev Argent Microbiol 2006;38:197-201.

31. Stanley T, Crothers L, McCalmont M, et al. The potential misidentification of Tsukamurella pulmonis as an atypical Mycobacterium species: a cautionary tale. J Med Microbiol 2006;55:475-8. 